\title{
Boron Difluoride Complex Dyes: Synthesis and Photophysical Properties
}

\author{
Meram S. Abdelrahman', Hussein A. Mohamed ${ }^{1, *}$ \\ ${ }^{1}$ Dyeing, Printing and Auxiliaries Department, Textile Industries Research Division, \\ National Research Centre, 33 El-Buhouth Street, Dokki, Cairo 12622, ${ }^{2}$ Central \\ Laboratories of the Egyptian Mineral Resources Authority, Cairo, Egypt
}

\begin{abstract}
RORON difluoride $\left(\mathrm{BF}_{2}\right)$ complex dyes are relatively non-polar and electrically neutral B tending to reduce the perturbation of the conjugate functional characteristics. Consequently, they are frequently the favored choice for biological labeling. Also, their conjugates of low molecular weight structures are more likely to be permeable to live cells than other conjugates of charged chromophores. As a result of their high extinction coefficient, another significant application of boron difluoride complexes is their reactivity and high detection ability to volatile amine reagents accessible for HPLC and electrophoresis. They are more useful than the majority of other dyes with long absorption wavelength. $\mathrm{BF}_{2}$ complex conjugates are extremely valuable reagents for many bio-analytical screening purposes, such as their affinity to quench the fluorescence upon conjugation to certain biopolymers. In this review, we discuss the preparation of boron difluoride carbonyl based-complexes, including O-O double-dentate and $\mathrm{N}-\mathrm{O}$ double-dentate dyes, as well as shedding light on their photophysical properties.
\end{abstract}

Keywords: Boron Difluoride; Complexe Dyes; Synthesis; photophysical properties.

\section{Introduction}

Functional dyestuffs, such as organic boron difluoride complexes, have been useful for hitech applications such as electronics, liquid crystalline displays, sensors, electrophotography, energy storage tools, security printing, optical data storage, organic light emitting devices, light control, solar cells and photodynamic therapy [1-11]. Functional dyestuffs are colorants that introduce a new function upon exposure to a small quantity of external energy, such as temperature, light, and magnetic/electric field. This external energy can control the electron-conjugated system leading to UV-Vis and/or emission changes, such as color change [12-24]. The chemistry of organic boron difluoride complexes has been recently received much more attention because of its significance for various practical applications [25]. Organic boron diffuoride materials are recognized as fluorescent molecules with high quantum yields and high fluorescence intensity [25]. Hence, they have been extensively used in various applications, such as photo-dynamic cancer cure, laser dyes, biological fluoro-probes, fluorescent indicators and photo-sensitizers. There are three main classes of organic boron difluoride ligands, including N-N double-dentate, N-O doubledentate and $\mathrm{O}-\mathrm{O}$ double-dentate ligands as shown in Figure 1 [22]. For the first two classes of boron difluoride complexes, both boradipyrromethene (BODIPY) and 1,3,2-dioxaborine are their corresponding representatives [26, 27]. Moreover, BODIPY based $\mathrm{BF}_{2}$ complexes have been investigated extensively because of their excellent fluorescence properties. However, there are low number of reports on $\mathrm{BF}_{2}$ complexes with $\mathrm{O}-\mathrm{O}$ double-dentate ligands, although the dioxaborine complexes have interesting fluorescence emission, first and second order non-linear optical properties, ion sensing ability, and can function as electron transport in organic emitting devices [28, 29]. In the relationship study between structure and photochemical properties; some reports demonstrated that annelation of electron donor to the dioxaborine ring resulted in improved absorption and fluorescence of the produced dyes and better resistance to hydrolysis [22, 25, 30]. 
<smiles>CCc1c(C)c2c(C)c3c(C)c(CC)n(P)n3c(C)c-2c1C</smiles>

(a) (b)

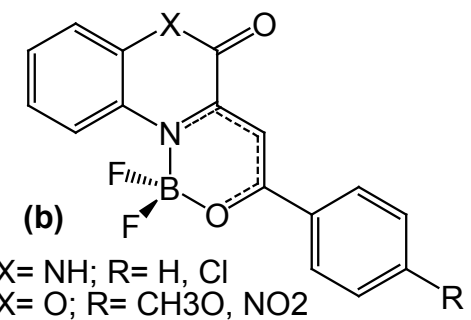<smiles></smiles>

Fig. 1. Main classes of organic boron difluoride dyes depending on their ligand structure, including N-N doubledentate (a), N-O double-dentate (b) and O-O double dentate (c) ligands.

The boron difluoride complex fluorophores comprise spectral properties that are frequently better than those of fluorescein, tetramethylrhodamine and other dyes with longer absorption wavelength. Boron difluoride complex dyes are generally used to produce fluorescent conjugates of different natural and biological polymers as well as the production of other fluorescent products, such as enzyme substrates, phospholipids and polystyrene microspheres [22-30].

$\mathrm{BF}_{2}$ dyes containing $\mathrm{O}-\mathrm{O}$ double-dentate ligands

A series of rigid $\beta$-diketone boron difluoride complexes have been prepared and used as good organo-gelators in some mixed solvents. The balanced $\pi-\pi$ interactions which can be tuned as a result of molecular conjugation, are the major key to the gel formation from rigid $\pi$-conjugated organic structures lacking alkyl chains and
H-bonding units. This approach offers a new perspective in designing new rigid organogelator systems and expanding the gel-phase material with attractive photonic and electronic utilities [30]. Self-assembly studies have been carried out on $\mathrm{BF}_{2}$-substituted $\mathrm{O}-\mathrm{O}$ double-dentate derivatives sowing spontaneous absorption and well-assembled two-dimensional lamellae. The H-bond among the hydrogen on ortho-carbon of the phenyl moiety and $\mathrm{BF}_{2}$ fluorine atom demonstrate a alongside configuration, while the H-bond among two carboxyl groups led to a pattern of molecules confronting each other. The outcomes have been noteworthy for designing and manufacturing various molecular assemblies of different molecular structures [31]. $\mathrm{BF}_{2}$ complexes of styryl $\beta$-diketone derivatives have also been reported (Figure 5) [32].<smiles>CN(C)c1ccc(/C=C/C(O)=C/C(=O)c2ccccc2)cc1</smiles><smiles></smiles>

Fig. 2. $\mathrm{BF}_{2}$ complex dyes with styryl $\beta$-diketone ligands. 
Dissimilar arrangements of tetraketone that have been prepared as described in Figure 3, demonstrate an improved side by side intermolecular reaction because of the complex formation. The studies on the three dimensional crystal structures dedicate the nature of the intermolecular interactions due to $\mathrm{H}$-bond formation. These intermolecular interactions are a significant feature fabricated by self-assembly [33].

Nanofibrils with a strong red emission produced from triphenylamine functionalized $\beta$-diketone- $\mathrm{BF}_{2}$ complexes. The emission of the nanofibrillated films can be quenched effectively and quickly when exposed to the vapors of organic amines (Figure 4), such as triethylamine, cyclohexylamine and butylamine. However, no emission quenching was detected for other ordinary reagents, such as dichloromethane, ethanol and toluene. The reported responsive time of these produced nanofibrillated films to aniline was about $1.06 \mathrm{~s}$, which is considered as one of the most rapid fluorescence response to aniline [34]. The elevated sensitivity, and short life and decay-time of the triphenylamine functionalized $\beta$-diketone- $\mathrm{BF}_{2}$ complexes for gaseous amines is due to the high surface area and large interspaces in the three dimensions network consisting of plenty of nanofibrils (prefer the improved adsorption and diffusion of amines vapors), as well as the improved emission quenching as a result of the improved intermolecular exciton diffusion alongside the long axis of one dimension nanostructures. The results were supportive to the design of new efficient fluorescence chemosensors in sensing traces of amines vapors [34].<smiles>CCOC(=O)C(=O)OCCNc1ccccc1/C(=C/C(=O)C(=O)/C=C(\O)c1ccccc1)OO</smiles>

Fig. 3. Synthesis of tetraketone-based BF2 complex dye.<smiles>[R]C1=COB(F)OC([R])=C1</smiles>

fluorescent

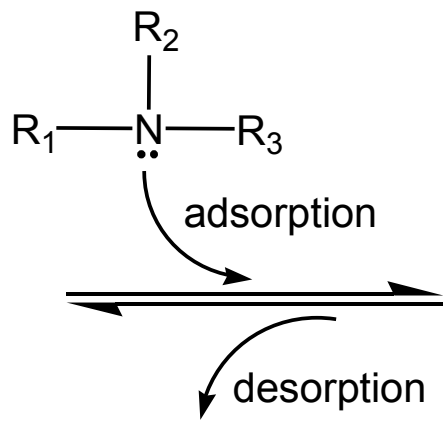<smiles>[R]N([R])[R]</smiles><smiles></smiles>

non-fluorescent

Fig. 4. Anticipated mechanism for the emission quenching upon exposure to volatile amines and its reversible process. 
A number of novel boron difluoride polymethine chromophores with low fluorescence have been synthesized to afford exceedingly emissive open-chain products, which upon heating cyclization give pyridone chromophores with poor fluorescence (Figure 5) [35].

Bis(dioxaborine) carbazole derivatives have been synthesized, and low dimensional nanostructured films with diverse morphologies have been constructed using carbon chains. These obtained nano-structured films afforded strong fluorescence displaying the ability to function as fluorescent chemo-sensors for monitoring aniline fumes, with dissimilar responsive rates and dissimilar sensitivity depending on their morphological properties that offer a large surface area for improved adsorption of vaporized molecules and allow expedient diffusion of that vaporized analytes (Figure 6) [36].

Bis(dioxaborine) fluorenes (Figure 7) have been reported as potential substrates for organic light-emitting diode (OLED) purposes [37]. There are various types of aromatic and heteroaromatic $\mathrm{BF}_{2}$ fluorophores bearing 1,3-diketone ligands have been reported as novel materials for lightemitting devices. Herein, the $\mathrm{BF}_{2}$-chelating fragments behaved as electron-accepting groups. The trifluoromethyl group introduced into the<smiles>CC1=C(/C=C/C=C2/N(C)c3ccccc3C2(C)C)c2c(cc(C)oc2=O)OB(F)O1</smiles><smiles>[R]N/C(C)=C\C1=C(C(=O)[O-])C(/C=C/C=C2/N(C)c3ccccc3C2(C)C)=C(/C=C/[NH3+])OB1F</smiles><smiles>[R]n1c(C)cc2c(c1=O)C(/C=C/C=C1/N(C)c3ccccc3C1(C)C)=C(C(C)C)OB(F)O2</smiles>

strong fluorescence

Fig. 5. Boron difluoride polymethine chromophore with low fluorescence with the ability to afford highly emissive open-chain chromophore, which upon heating cyclize to give pyridone with poor fluorescence.

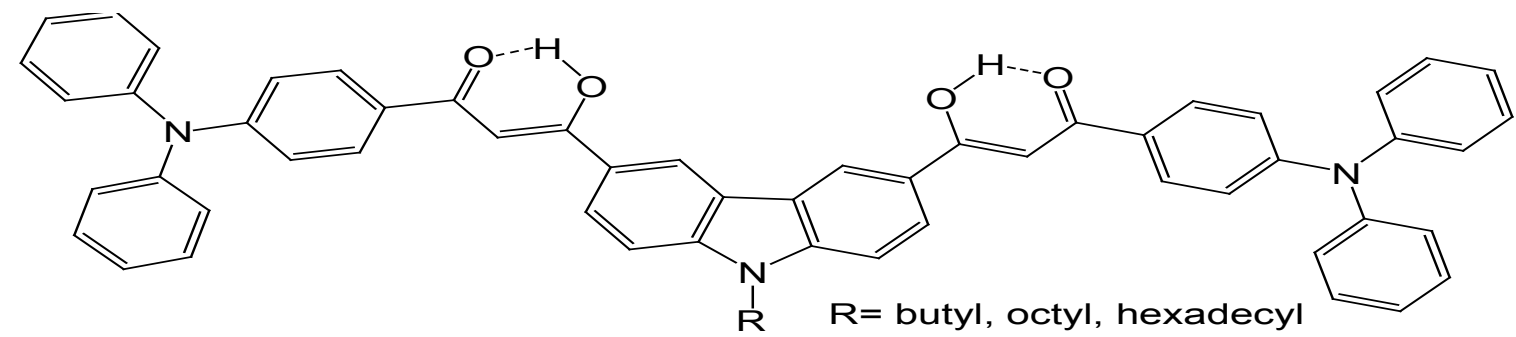

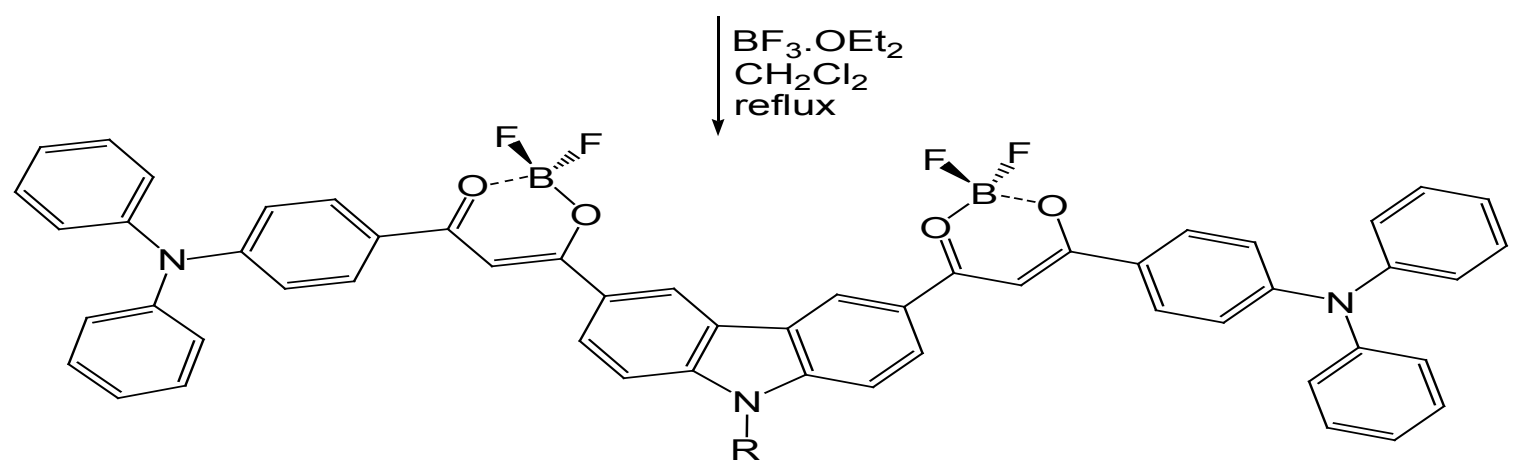

Fig. 6. Synthesis of bis(dioxaborine) carbazole complex dyes. 

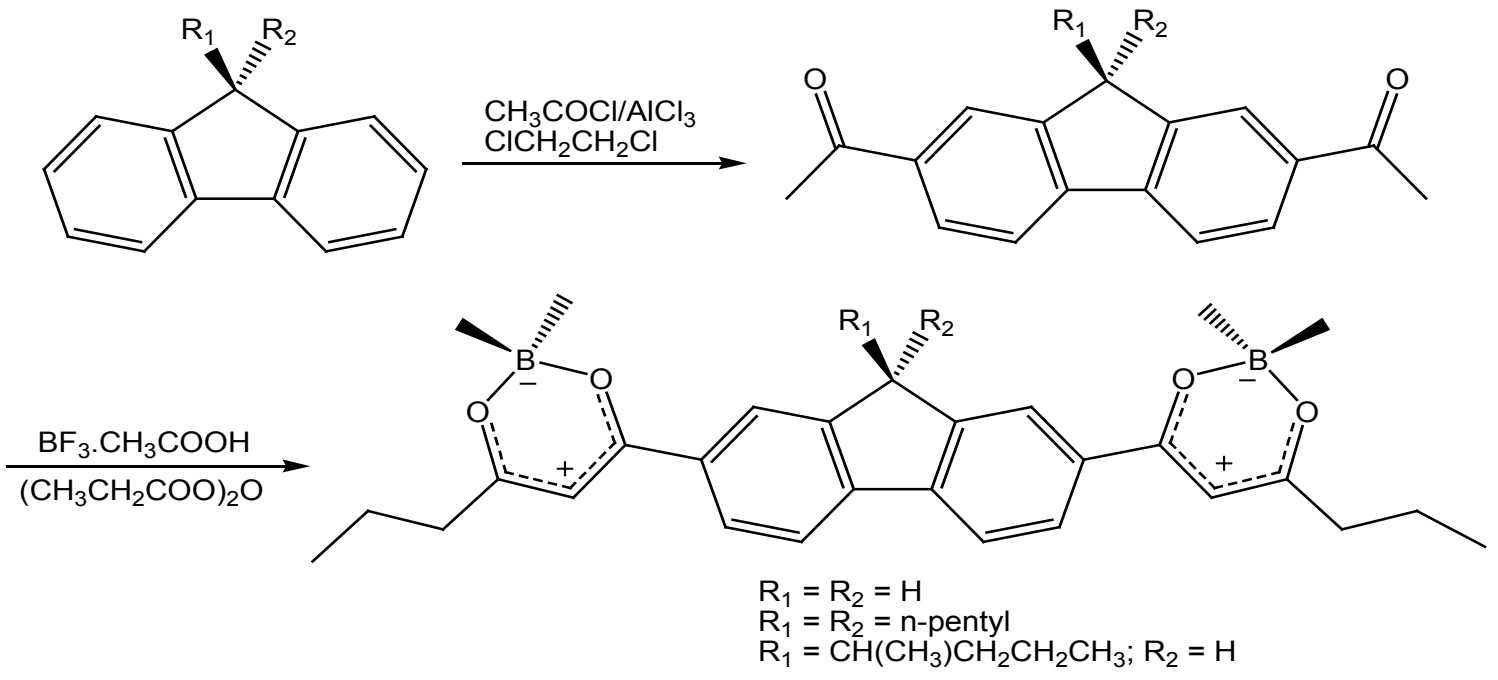

Fig. 7. Synthesis of bis(dioxaborine) fluorenes.

$\mathrm{BF}_{2}$-chelating fragment to amplify the electron affinity. The anion state of the produced $\mathrm{BF}_{2}$ complexes were stabilized as a result of the 1,3-diaryl-1,3-diketone ligands [38].

Doubly bridged boron difluoride complexes (Figure 8) with oxalyl-tethered pyrrole ligands have been reported to afford bright orange chromophores acting as a well discriminating chromogenic sensor for both fluoride and cyanide ions. Adding fluoride and cyanide ions into polar organic solvents containing doubly bridged boron difluoride complexe dye, led to a reversible detachment of the $\mathrm{BF}_{2}$ bridges affording sharp variations in color $[39,40]$.

A supramolecular organogelators with $\pi$-conjugated binding sites have been prepared as anion responsive $\mathrm{BF}_{2}$ complex dyes of dipyrrolyldiketones or known as 1,3-dipyrrolyl1,3-propanedione boron complexes to function as competent acyclic anion responsive materials through the inversion of pyrrole moieties [41]. The anion binding behavior was controlled by modifying the periphery of the receptors to afford supramolecular organogelators based on $\pi-\pi$ and van-der-waals interactions $[42,43]$. The synthesis of $\mathrm{BF}_{2}$ complex derivatives starting from pyrrolyldiketones is described in Figure 9.

Another class of anion responsive materials based on $\mathrm{BF}_{2}$-based complexes of 1,3-dipyrrolyl1,3-propanedione fluorinated derivatives (Figure 10), have been reported as effectively binding anions. Hence, more modification in the acyclic receptor will recognize the binding and transporting of such biotic anion species, including proteins and nucleotides, in water [44-46]. $\mathrm{BF}_{2}$ complexes of 3 -acyl tetramic acid derivatives was prepared by the acylation of pyrrolidine-2,4-diones with acid chlorides and $\mathrm{BF}_{3}$-etherate [47].

Solutions of alkyl-substituted $\mathrm{BF}_{2}$ complexes have a green fluorescein-like fluorescence. Nevertheless, substituents that produce extra conjugation can shift the absorption and emission spectra to considerably longer wavelengths. Two dialkylaminogroupsonthecoumarincorecomplexto 2, 2-difluoro-1,3,2-dioxaborine moiety remarkably decreased the electron withdrawing capacity of the dioxaborine fragment. As a consequence, the spectral luminescent characteristics changed in going from a diethylamino group to a dipyrrolidino group. This alteration in the dye construction also led to considerably amplified resistance to hydrolysis [48]. 2-Aminochromones was also prepared by reacting phosgene iminium salts with 2'-hydroxyacetophenone- $\mathrm{BF}_{2}$ complexes to afford $\beta$-chlorovinylogous amide $\mathrm{BF}_{2}$ complex derivatives [49]. $\mathrm{BF}_{2}$ complexes based on perylene and tetracene (Figure 11) ligands were prepared as novel category of electron deficient arenes with long wavelength absorption and better electron affinity because of the quadrupolar structures corresponding to the resonance contributors. Tetracene-based $\mathrm{BF}_{2}$ complexes are n-type semiconductor [50].

A boron difluoride complex containing dihydroxydione of octafluorotetracene fragment was reported as a novel class of electron accepting materials revealing long wavelength absorption depending on the perfluorotetracene

J. Text. Color. Polym. Sci. 17, No. 1 (2020) 
<smiles>O=C(Cl)C(=O)Cl</smiles>
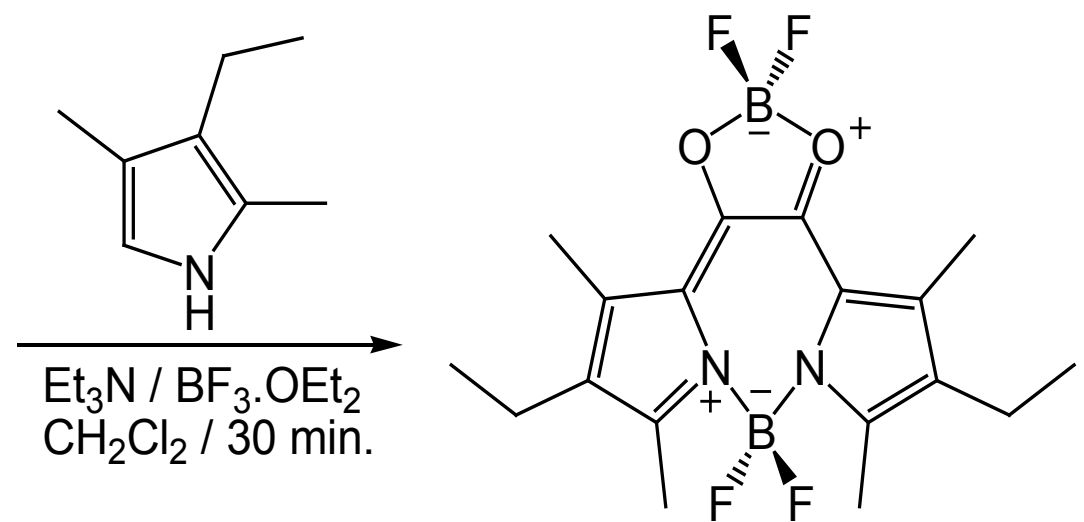

Fig. 8. Synthesis of doubly bridged BF2 complex.<smiles></smiles>

Fig. 9. Synthesis of BF2 complex derivatives from pyrrolyldiketones.<smiles>[X]c1[nH]c(C(=O)CC(=O)c2[nH]c([R])c([X])c2[X])c([X])c1[X]</smiles>

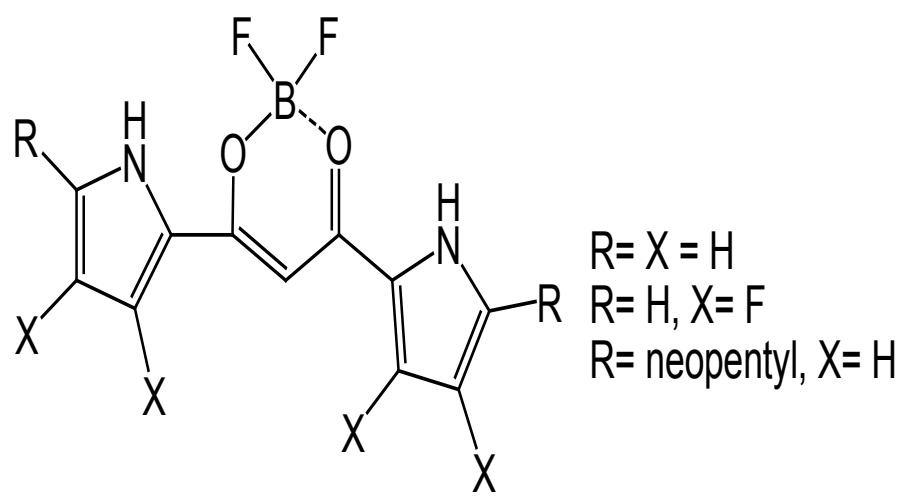

Fig. 10. Dipyrrolyldiketone derivatives and their BF2 complexes. 
<smiles>FB1Oc2c3c(c4ccccc4c-3c3c(c4ccccc24)O[As](F)(F)O3)O1</smiles><smiles></smiles><smiles>F[B-]1(F)Oc2ccc3c4ccc5c6c(ccc(c7ccc(c2c37)O1)c64)O[B-](F)(F)O5</smiles><smiles>F[B-]1(F)Oc2ccc3c4ccc5c6c(ccc(c7ccc(c2c37)O1)c64)O[B-](F)(F)O5</smiles>

Fig. 11. Molecular structures of perylene and tetracene BF2 complex dyes.

frame and elevated electron affinities owing to its quadrupolar configuration improved by fluorination [51]. Another novel boron difluoride complexes functionalized with a pyrene ligand have been reported as binuclear boron difluoride complexes for OLED. In the solid-state, they displayed strong red fluorescence. Compared to conventional emission and electron transport materials, additional structural optimization improving the emission intensity in the solid state is likely to afford more efficient emission materials [35]. Reacting $\mathrm{BF}_{3}$-etherate with 3-acetyl-4-hydroxy-1-methyl-2-quinolone (Figure 12) afforded the corresponding $\mathrm{BF}_{2}$ complex which was subjected to a condensation reaction with a range of carbonyl compounds to produce polymethine and styryl 2-quinolone derivatives having efficient fluorescence and intense absorption in the visible region of the electromagnetic radiation. The formed $\mathrm{BF}_{2}$ complex substantially enhances the reactivity of the methyl group of the acetyl fragment [52].

Boron difluoride O-O double-dentate complexes with a push-pull structural design, in which a pyrazoline-1,3-dione is the electron donor; have been prepared. These new type of pyrazoline1,3-dione based-complexes demonstrated photophysical properties that are extremely reliant to the solvent polarity and aggregation conditions. The molecular assembly of the crystal structure resulted in physically powerful charge transfer characterized with an extended excited state lifetime [53]. Reacting those 2-formyl ketone-based boron difluoride complexes with organometallic reagents has been reported to comprise an efficient preparation of 2-alkylidene ketones. Reacting Grignard reagents with 2-formyl ketones was revealed to be an efficient process for the preparation of 2-alkylidene ketones [54]. Chemoselective reaction of organolithium reagents with a boron difluoride complex of 3 -oxopropanoates has been reported as shown in Figure 13. This attitude permits straightforward synthesis of different 1,3-dioxa-boron difluoride complexes and consequently led to the creation of asymmetrically substituted 1,3-diketones from their corresponding 3-oxopropanoate derivatives $[55,56]$. 
$\mathrm{BF}_{2}$ dyes containing $\mathrm{N}-\mathrm{O}$ double-dentate ligands

It has been reported the preparation and X-ray crystallography of $\mathrm{BF}_{2}$ complex from N-O doubledentate ligands originated from Schiff's bases of salicylaldehyde. However, its low emission intensity and quantum yield forced researchers to investigate for further methods toward such complex materials. 1,3-Enaminoketone derivatives are iso-steric analogues to the corresponding 1,3-enolic ketones, and are outstanding entrants as N-O double-dentate ligands to produce 1,3,2-oxazaborines, which are believed to exhibit photophysical characteristics comparable to those of 1,3,2-dioxaborines derived from 1,3-diketones [57]. New types of fluorescent dyes based on 2-ketopyrrole $\mathrm{BF}_{2}$ Complexes have been synthesized. BODIPY dyes are precious molecular labels due to their intense fluorescence characteristics. The aryl substituted complexes $\mathrm{N}-\mathrm{O}$ double-dentate ligands are fluorescent compounds that tend to emit at lower wavelengths than the analogous BODIPY dyes; N-N doubledentate ligands. These aryl substituted N-O double- dentate complexes have a reduced fluorescence thus demonstrating the significance of the added, constrained, aromatic moiety in these structures [58]. New $\mathrm{BF}_{2}$ complexes of 3-methylthio-enaminones have been prepared efficiently by the reaction of 3-methylthio-enaminones with $\mathrm{BF}_{3} \cdot \mathrm{Et}_{2} \mathrm{O}$ in the existence of $\mathrm{Et}_{3} \mathrm{~N}$ (Figure 14). These new materials are supportive for better understanding of chemical reactivity of enaminones and might be significant in pharmaceutical and agrochemical purposes [59].

Two types of new boron difluoride complexes with arylethylidene-quinoxalin-2-ones or (arylethylidene)-benzo[1,4] oxazin-2-ones as their heterocyclic 1,3-enaminoketones (Figure 15). The chelation process can be achieved readily and efficiently to give the targeted complexes in high yields. For those boron difluoride complexes, the large span red-shifts of UV-Vis absorption and intense emission originated from the substantial conjugated systems among the aromatic cycles and the chelating moieties [26].<smiles></smiles>

Fig. 12. Synthesis of 3-acetyl-4-hydroxy-1-methyl-2-quinolone BF2 complex.

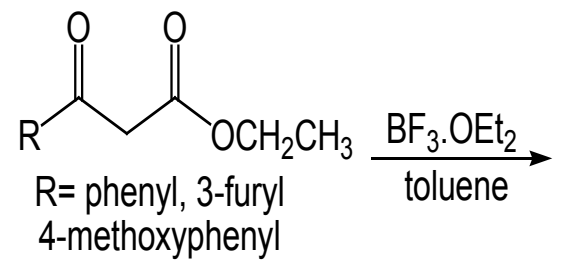<smiles></smiles>

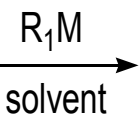<smiles></smiles>

Fig. 13. Reaction of boron difluoride complexes of ethyl-3-oxopropanoates with organolithium reagent (R1M).

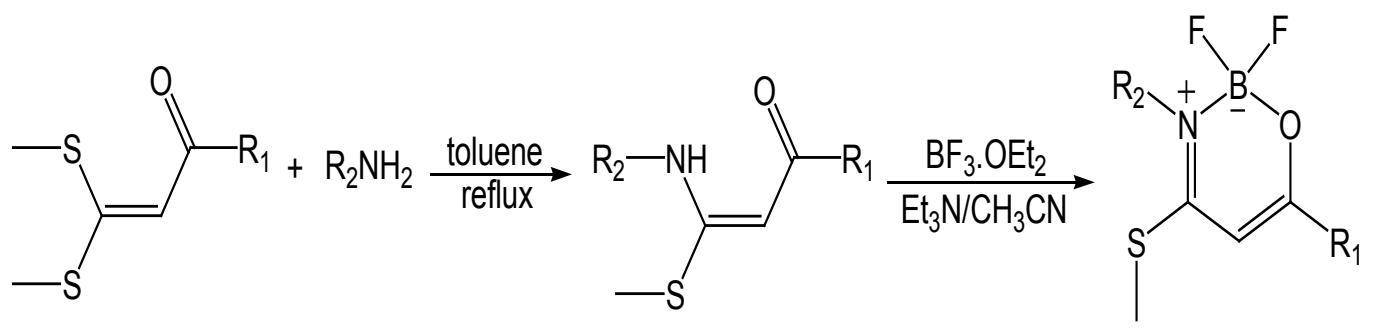

Fig. 14. Synthesis of BF2 complex using 3-methylthio-enaminone. 


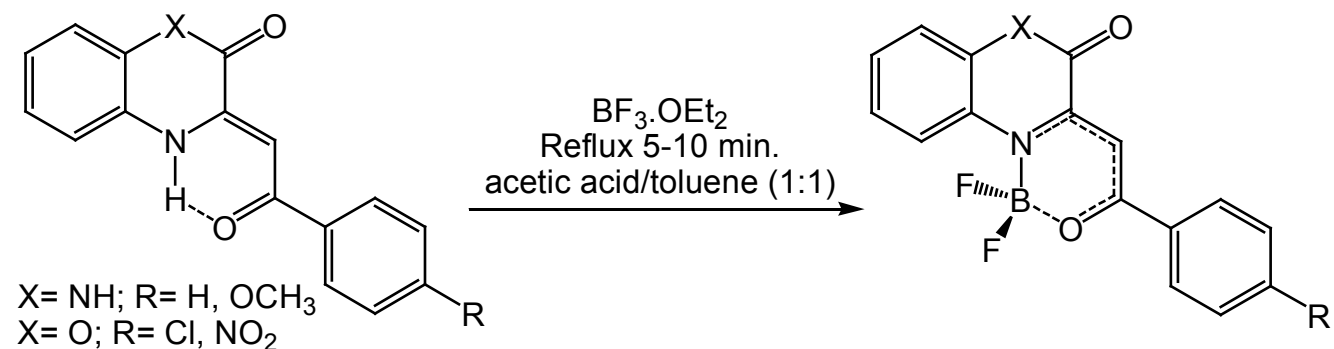

Fig. 15. Synthesis of boron difluoride complex derivatives containing arylethylidene-quinoxalin-2-ones or (arylethylidene)-benzo[1,4]oxazin-2-ones.

\section{Conclusion}

Boron difluoride organic complexes with pushpull structures have been highly considered because of the well-known electron-acceptor $\mathrm{BF}_{2}$ and their ability to accept the negative charge partially from the electron-donor using delocalized- $\pi$-bridge systems. Mainly, there are three different classes of boron difluoride organic complexes, known as $\mathrm{O}-\mathrm{O}$ double dentate, $\mathrm{N}-\mathrm{O}$ double-dentate and $\mathrm{N}-\mathrm{N}$ double-dentate materials depending on the ligand type. For the earlier two types of boron difluoride complexes, boradipyrromethene and 1 , 3, 2-dioxaborine are their equivalent analogues. In addition, boron dipyrromethene derivatives have been considered extensively owing to their excellent emissive characteristics. Nevertheless, there are few reports accessible on the production of boron difluoride complexes based on O-O double-dentate ligands, although dioxaborine complexes have outstanding photophysical behavior. For example, those compounds show attractive fluorescence, first and second-order nonlinear optical behavior, ion responsive capability, and can provide fluorescent and electron transport films in organic light emitting devices.

\section{References}

1. Shan Wang, Ji-Ting Hou, Mei-Lin Feng, XiaoZhuan Zhang, Shan-Yong Chen, and Xiao-Qi Yu. "Cobalt (III)-catalyzed alkenylation of arenes and 6-arylpurines with terminal alkynes: efficient access to functional dyes." Chemical Communications 52, no. 13, 2709-2712 (2016).

2. Tawfik A. Khattab, Moustafa MG Fouda, Meram S. Abdelrahman, Sarah I. Othman, May BinJumah, Maha Abdulla Alqaraawi, Haifa Al Fassam, and Ahmed A. Allam. "Development of illuminant glow-in-the-dark cotton fabric coated by luminescent composite with antimicrobial activity and ultraviolet protection." Journal of fluorescence 29, no. 3, 703-710 (2019).

3. Juraj Bujdák. "Hybrids with functional dyes" In Inorganic Nanosheets and Nanosheet-Based Materials, pp. 419-465. Springer, Tokyo, (2017).

4. Jiajun Wu, Dezhi He, Li Zhang, Yudong Liu, Xiaogang Mo, Jianbin Lin, and Hui-jun Zhang. "Direct synthesis of large-scale ortho-iodinated perylene diimides: key precursors for functional dyes." Organic letters 19, no. 19, 5438-5441 (2017).

5. Tawfik A. Khattab, Moustafa MG Fouda, Meram S. Abdelrahman, Sarah I. Othman, May BinJumah, Maha Abdulla Alqaraawi, Haifa Al Fassam, and Ahmed A. Allam. "Co-encapsulation of enzyme and tricyanofuran hydrazone into alginate microcapsules incorporated onto cotton fabric as a biosensor for colorimetric recognition of urea." Reactive and Functional Polymers 142, 199-206.

6. Meram S. Abdelrahman, and Tawfik A. Khattab. "Development of One-Step Water-Repellent and Flame-Retardant Finishes for Cotton." Chemistry Select 4, no. 13, 3811-3816 (2019).

7. Meram S. Abdelrahman, Sahar H. Nassar, Hamada Mashaly, Safia Mahmoud, Dalia Maamoun, Mohamed El-Sakhawy, Tawfik A. Khattab, and Samir Kamel. "Studies of Polylactic Acid and Metal Oxide Nanoparticles-Based Composites for Multifunctional Textile Prints." Coatings 10, no. 1, 58 (2020).

8. Meram S. Abdelrahman, Sahar H. Nassar, Hamada Mashaly, Safia Mahmoud, Dalia Maamoun, and Tawfik A. Khattab. "Polymerization products of lactic acid as synthetic thickening agents for textile printing." Journal of Molecular Structure 1203, 127421 (2020).

J. Text. Color. Polym. Sci. 17, No. 1 (2020) 
9. Tawfik A. Khattab, Meram S. Abdelrahman, and Mohamed Rehan. "Textile dyeing industry: environmental impacts and remediation." Environmental Science and Pollution Research: 1-16.

10. Takeshi Maeda, Jin Zhou, Yuya Oda, Hiroyuki Nakazumi, and Shigeyuki Yagi. "Synthesis and properties of functional dyes with squarainenaphthalene diimide hybrid structure." Research on Chemical Intermediates 44, no. 8, 4783-4795 (2018).

11. Meram S. Abdelrahman, Moustafa MG Fouda, Jamaan S. Ajarem, Saleh N. Maodaa, Ahmed A. Allam, and Tawfik A. Khattab. "Development of colorimetric cotton swab using molecular switching hydrazone probe in calcium alginate." Journal of Molecular Structure , 128301 (2020).

12. Meram Abdelrahman, Sahar Wahab, Hamada Mashaly, Dalia Maamoun, and Tawfik A. Khattab. "Review in Textile Printing Technology." Egyptian Journal of Chemistry (2020). DOI: 10.21608/ ejchem.2020.23726.2418.

13. Tawfik A. Khattab, Mahmoud Abd El-Aziz, Meram S. Abdelrahman, Manal El-Zawahry, and Samir Kamel. "Development of long-persistent photoluminescent epoxy resin immobilized with europium (II)-doped strontium aluminate." Luminescence 35, no. 4, 478-485 (2020).

14. Sherif Abdelmoez, Meram Abdelrahman, and Tawfik Khattab. "Synthesis, Solvatochromic Properties and pH Sensory of Novel Symmetrical Bis (Tricyanofuran) hydrazone Chromophore." Egyptian Journal of Chemistry 62, no. 7, 1197-1206 (2019).

15. María M. Alcaide, Fabio MF Santos, Vânia F. Pais, Joana Inês Carvalho, Daniel Collado, Ezequiel Pérez-Inestrosa, Jesús F. Arteaga, Francisco Boscá, Pedro MP Gois, and Uwe Pischel. "Electronic and functional scope of boronic acid derived salicylidenehydrazone (BASHY) complexes as fluorescent dyes." The Journal of organic chemistry 82, no. 14, 7151-7158 (2017).

16. Tawfik A. Khattab, Sawsan Dacrory, Hussein Abou-Yousef, and Samir Kamel. "Development of microporous cellulose-based smart xerogel reversible sensor via freeze drying for nakedeye detection of ammonia gas." Carbohydrate polymers 210, 196-203 (2019).

17. Christopher Farley, Amit Aggarwal, Sunaina Singh, Aaron Dolor, Philip To, Alexander Falber, Maxwell Crossley, and Charles Michael Drain. "A Structural Model of Nitro-Porphyrin Dyes Based on Spectroscopy and Density Functional Theory."

J. Text. Color. Polym. Sci. 17, No. 1 (2020)
Journal of computational chemistry 39, no. 18 , 1129-1142 (2018).

18. Tawfik A. Khattab. "Novel solvatochromic and halochromic sulfahydrazone molecular switch." Journal of Molecular Structure 1169, 96-102 (2018).

19. Katharine L. Dobson, Carmel L. Howe, Yuri Nishimura, and Vincenzo Marra. "Dedicated setup for the photoconversion of fluorescent dyes for functional electron microscopy." Frontiers in cellular neuroscience 13, 312 (2019).

20. Rifaat Hilal, Saadullah G. Aziz, Osman I. Osman, and Jean-Luc Bredas. "Time dependent-density functional theory characterization of organic dyes for dye-sensitized solar cells." Molecular Simulation 43, no. 18, 1523-1531 (2017).

21. Tawfik A. Khattab, Ahmed M. Gabr, Ayman M. Mostafa, and Tamer Hamouda. "Luminescent plant root: a step toward electricity-free natural lighting plants." Journal of Molecular Structure 1176, 249-253 (2019).

22. Hui-hui Zhang, Xiong Hu, Wei Dou, and Weisheng Liu. "Photoluminescence properties of new BF2 complexes with pyrazolone ligands: Dependence on volume and electronic effect of substituents." Journal of Fluorine Chemistry 131, no. $8,883-887$ (2010).

23. Bing Tang, Fan Lv, Kangkang Chen, Lijuan Jiao, Qingyun Liu, Hua Wang, and Erhong Hao. "Development of BODIPY dyes with versatile functional groups at 3, 5-positions from diacyl peroxides via $\mathrm{Cu}$ (ii)-catalyzed radical alkylation." Chemical communications 55, no. 32 (2019): 4691-4694.

24. Tawfik A. Khattab, Hussein Abou-Yousef, and Samir Kamel. "Photoluminescent spray-coated paper sheet: Write-in-the-dark." Carbohydrate polymers 200,154-161 (2018).

25. Zhijian Chen, Yong Liu, Wolfgang Wagner, Vladimir Stepanenko, Xiangkui Ren, Soichiro Ogi, and Frank Würthner. "Near-IR Absorbing J-Aggregate of an Amphiphilic BF2Azadipyrromethene Dye by Kinetic Cooperative Self-Assembly." Angewandte Chemie International Edition 56, no. 21, 5729-5733 (2017).

26. Min Xia, Bin Wu, and Guofeng Xiang. "Synthesis, structure and spectral study of two types of novel fluorescent BF2 complexes with heterocyclic 1, 3-enaminoketone ligands." Journal of Fluorine Chemistry 129, no. 5, 402-408 (2008). 
27. Charles M. Lukehart, and LouAnn Sacksteder. "Diels-Alder reactions utilizing (ferrabeta.-diketonato) BF2 complexes containing alkynyl substituents as activated dienophiles." Organometallics 6, no. 1, 150-152 (1987).

28. Nicholas A. Ayoub, Amberle R. Browne, Bryce L. Anderson, and Thomas G. Gray. "Cyclometalated gold (III) trioxadiborrin complexes: studies of the bonding and excited states." Dalton Transactions 45, no. 9, 3820-3830 (2016).

29. Xiaojie Ren, Fan Zhang, Hongchen Luo, Lide Liao, Xiangzhi Song, and Wenqiang Chen. "Red-emitting Boron Difluoride Complexes with Mega-large Stokes Shift and Unexpectedly High Fluorescent Quantum Yield." Chemical Communications (2020).

30. Xiaofei Zhang, Ran Lu, Junhui Jia, Xingliang Liu, Pengchong Xue, Defang Xu, and Huipeng Zhou. "Organogel based on $\beta$-diketone-boron difluoride without alkyl chain and H-bonding unit directed by optimally balanced $\pi-\pi$ interaction." Chemical Communications 46, no. 44, 8419-8421 (2010).

31. Xu Zhang, Cun-Ji Yan, Ge-Bo Pan, Rui-Qin Zhang, and Li-Jun Wan. "Effect of C- H---F and $\mathrm{O}-\mathrm{H}-\mathrm{-}-\mathrm{O}$ Hydrogen Bonding in Forming Self-Assembled Monolayers of BF2-Substituted $\beta$-Dicarbonyl Derivatives on HOPG: STM Investigation." The Journal of Physical Chemistry C 111, no. 37, 13851-13854 (2007).

32. K.Gustav,U.Bartsch, andW.Guenther., Spectroscopic Studies on Organic Carbonyl Compounds, X: Comparative Absorption, Fluorescence, and NMR Measurements on Selected -Diketones and their BF 2-and Be-Complexes." Monatshefte fur Chemie 125, no. 12, 1321-1321 (1994).

33. Alexander O. Terent'ev, Ivan A. Yaremenko, Vera A. Vil, Valery M. Dembitsky, and Gennady I. Nikishin. „Boron trifluoride as an efficient catalyst for the selective synthesis of tricyclic monoperoxides from $\beta, \delta$-triketones and $\mathrm{H} 2 \mathrm{O} 2$.“ Synthesis 45, no. 02, 246-250 (2013).

34. Xiaofei Zhang, Xingliang Liu, Ran Lu, Haojun Zhang, and Peng Gong. "Fast detection of organic amine vapors based on fluorescent nanofibrils fabricated from triphenylamine functionalized $\beta$-diketone-boron difluoride." Journal of Materials Chemistry 22, no. 3, 1167-1172 (2012).

35. Andriy O. Gerasov, Mykola P. Shandura, Yuriy P. Kovtun, Yuriy G. Vlasenko, and Vasyliy F. Gorchev. "1, 3, 2-(2H)-dioxaborine polymethine dyes on the base of dehydroacetic acid-effective fluorescent amine probes." Journal of Heterocyclic Chemistry 45, no. 6, 1665-1672 (2008).

36. Xingliang Liu, Xiaofei Zhang, Ran Lu, Pengchong Xue, Defang $\mathrm{Xu}$, and Huipeng Zhou. „Lowdimensional nanostructures fabricated from bis (dioxaborine) carbazole derivatives as fluorescent chemosensors for detecting organic amine vapors." Journal of Materials Chemistry 21, no. 24, 8756-8765 (2011).

37. Benoit Domercq, Cara Grasso, Jose-Luis Maldonado, Marcus Halik, Stephen Barlow, Seth R. Marder, and Bernard Kippelen. "Electrontransport properties and use in organic lightemitting diodes of a bis (dioxaborine) fluorene derivative." The Journal Of Physical Chemistry B 108, no. 25, 8647-8651 (2004).

38. Katsuhiko Ono, Kyohei Yoshikawa, Yujiro Tsuji, Hiroyuki Yamaguchi, Rie Uozumi, Masaaki Tomura, Keijiro Taga, and Katsuhiro Saito. "Synthesis and photoluminescence properties of BF2 complexes with 1, 3-diketone ligands." Tetrahedron 63, no. 38, 9354-9358 (2007).

39. Ruslan Guliyev, Seyma Ozturk, Ertan Sahin, and Engin U. Akkaya. "Expanded bodipy dyes: anion sensing using a bodipy analog with an additional difluoroboron bridge." Organic letters 14, no. 6, 1528-1531 (2012).

40. Todd W. Hudnall, Tzu-Pin Lin, and François P. Gabbaï. "Substitution of hydroxide by fluoride at the boron center of a BODIPY dye." Journal of Fluorine Chemistry 131, no. 11, 1182-1186 (2010).

41. Hiromitsu Maeda, Mayumi Takayama, Kazuki Kobayashi, and Hideyuki Shinmori. "Modification at a boron unit: tuning electronic and optical properties of $\pi$-conjugated acyclic anion receptors." Organic \& biomolecular chemistry 8, no. 19, 4308-4315 (2010).

42. Hiromitsu Maeda, and Yohei Haketa. "Selective iodinated dipyrrolyldiketone BF 2 complexes as potential building units for oligomeric systems." Organic \& biomolecular chemistry 6, no. 17, 3091-3095 (2008).

43. Chikoto Fujimoto, Yukio Kusunose, and Hiromitsu Maeda. "CH---Anion Interaction in BF2 Complexes of C3-Bridged Oligopyrroles." The Journal of organic chemistry 71, no. 6, 2389-2394 (2006).

44. Hiromitsu Maeda, and Yoshihiro Ito. "BF2 complex of fluorinated dipyrrolyldiketone: a new 
class of efficient receptor for acetate anions." Inorganic chemistry 45, no. 20, 8205-8210 (2006).

45. Hiromitsu Maeda, Yukio Kusunose, Yuta Mihashi, and Tadashi Mizoguchi. "BF2 Complexes of $\beta$-Tetraethyl-Substituted Dipyrrolyldiketones as anion receptors: potential building subunits for oligomeric systems." The Journal of organic chemistry 72, no. 7, 2612-2616 (2007).

46. Hiromitsu Maeda, Yohei Haketa, Yuya Bando, and Shohei Sakamoto. "Synthesis, properties, and solid-state assemblies of $\beta$-alkyl-substituted dipyrrolyldiketone BF2 complexes." Synthetic metals 159, no. 9-10, 792-796 (2009).

47. Raymond CF Jones, and Graeme E. Peterson. "Acylation of pyrrolidine-2, 4-diones: boron difluoride complexes of 3-acyl tetramic acids." Tetrahedron letters 24, no. 43, 4757-4760 (1983).

48. Andrii O. Gerasov, Konstantin V. Zyabrev, Mykola P. Shandura, and Yuriy P. Kovtun. "The structural criteria of hydrolytic stability in series of dioxaborine polymethine dyes." Dyes and Pigments 89, no. 1, 76-85 (2011).

49. Joel Morris, Yue Fang, Donn G. Wishka, and Fusen Han. "Synthesis and reactivity of boron difluoride complexes of N, N-dimethylsalicylacetamide." Tetrahedron letters 34, no. 24, 3817-3820 (1993).

50. Katsuhiko Ono, Hiroyuki Yamaguchi, Keijiro Taga, Katsuhiro Saito, Jun-ichi Nishida, and Yoshiro Yamashita. "Synthesis and properties of BF2 complexes to dihydroxydiones of tetracene and perylene: novel electron acceptors showing n-type semiconducting behavior." Organic letters 11, no. 1,149-152 (2009).

51. Katsuhiko Ono, Junko Hashizume, Hiroyuki Yamaguchi, Masaaki Tomura, Jun-ichi Nishida, and Yoshiro Yamashita. "Synthesis, crystal structure, and electron-accepting property of the BF2 complex of a dihydroxydione with a perfluorotetracene skeleton." Organic letters 11, no. $19,4326-4329$ (2009).

52. A. V. Manaev, I. N. Okhrimenko, and K. A. Lyssenko. "Synthesis and condensation reactions of the boron difluoride complex with 3-acetyl4-hydroxy-1-methyl-2-quinolone."Russian Chemical Bulletin, 57, no. 8, 1734-1739 (2008).

53. S. A. Kon'kov, and I. K. Moiseev. "Synthesis of pyrazoles and pyrazolones from 1,3-and 1,4-diketones of the adamantane series." Russian journal of organic chemistry 45, no. 12, 1824-1828 (2009).
54. Robin AJ Smith, and Thomas A. Spencer. "Synthesis of 2-alkylidene ketones. Chemistry of boron difluoride complexes of 2-formyl ketones." The Journal of Organic Chemistry 35, no. 10, 3220-3223 (1970).

55. O. Altan Bozdemir, Sundus Erbas-Cakmak, O. Oner Ekiz, Aykutlu Dana, and Engin U. Akkaya. "Towards Unimolecular Luminescent Solar Concentrators: Bodipy-Based Dendritic Energy-Transfer Cascade with Panchromatic Absorption and Monochromatized Emission." Angewandte Chemie International Edition 50, no. 46, 10907-10912 (2011).

56. Bogdan Stefane. "Selective addition of organolithium reagents to BF2-chelates of $\beta$-ketoesters." Organic letters 12, no. 13, 2900-2903 (2010).

57. Jiong Chen, Armin Burghart, Chi-Wai Wan, Long Thai, Cesar Ortiz, Joe Reibenspies, and Kevin Burgess. "Synthesis and spectroscopic properties of 2-ketopyrrole-BF2 complexes: a new class of fluorescent dye." Tetrahedron Letters 41, no. 14, 303-2307 (2000).

58. Martin Bröring, Robin Krüger, and Christian Kleeberg. "BF2-Chelate Complexes of 6-(4-Iodophenyl)-2, 3, 4, 8, 9, 10-hexamethyldipyrrin and 2-(4-Iodobenzoyl)-3, 4, 5-trimethylpyrrole: Fluorescent Dyes with a Chemical Anchor Group." Zeitschrift für anorganische und allgemeine Chemie $\mathbf{6 3 4}$, no. 9, 1555-1559 (2008).

59. Ti Zhang, Yue-Mei Jia, Sheng-Jiao Yan, Chu-Yi $\mathrm{Yu}$, and Zhi-Tang Huanga. "Synthesis of BF2 complex of 3-methylthio enaminones." Arkivoc 14, 156-170 (2009).

(Received $28 / 5 / 2020$ accepted $6 / 8 / 2020$ 


\section{أصباغ مركب البورون ثنائي فلوريد: طرق التحضير والخصائص الفيزيائية الضوئية

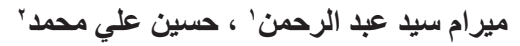

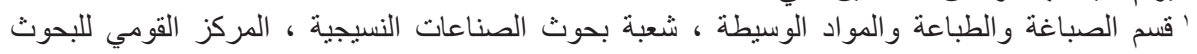

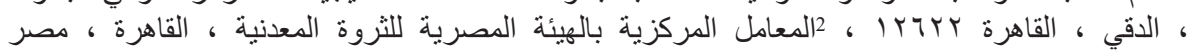

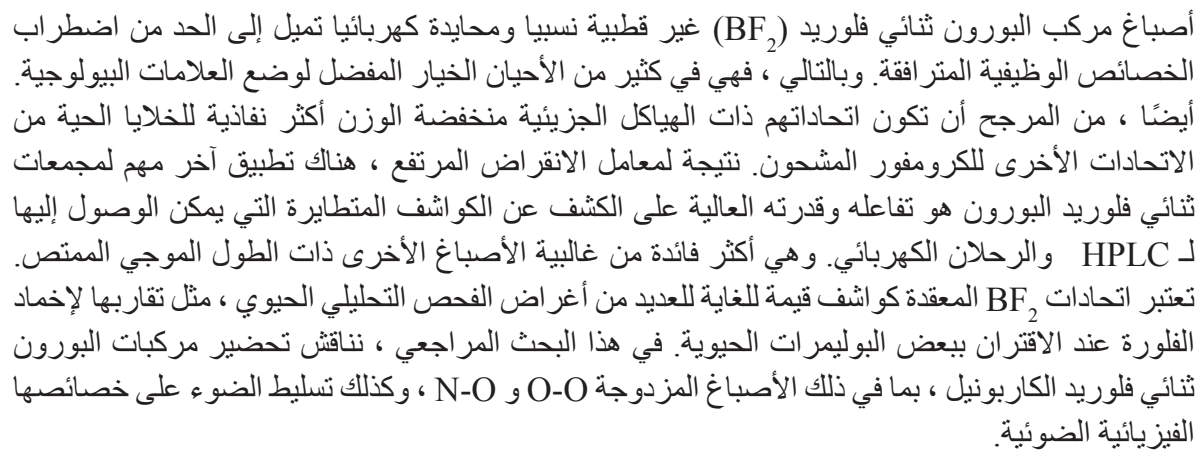

